\title{
VHL genetic alteration in CCRCC does not determine de-regulation of HIF, CAIX, hnRNP A2/B1 and osteopontin
}

\author{
Michelle J. Nyhan a, Shereen M. El Mashad ${ }^{\text {a }}$, Tracey R. O’Donovan ${ }^{\text {a }}$, Sarfraz Ahmad ${ }^{\mathrm{a}, \mathrm{b}}$, \\ Chris Collins ${ }^{a}$, Paul Sweeney ${ }^{b}$, Eamonn Rogers ${ }^{b}$, Gerald C. O’Sullivan ${ }^{\text {a,b, }}{ }^{* *}$ and \\ Sharon L. McKenna ${ }^{\mathrm{a}, *, * *}$ \\ ${ }^{a}$ Leslie C. Quick Laboratory, Cork Cancer Research Centre, BioSciences Institute, University College Cork and \\ Mercy University Hospital, Cork, Ireland \\ ${ }^{\mathrm{b}}$ Department of Surgery, Mercy University Hospital, Cork, Ireland
}

\begin{abstract}
Background: von Hippel-Lindau (VHL) tumour suppressor gene inactivation is associated with clear cell renal cell carcinoma (CCRCC) development. The VHL protein (pVHL) has been proposed to regulate the expression of several proteins including Hypoxia Inducible Factor- $\alpha$ (HIF- $\alpha$ ), carbonic anhydrase (CA)IX, heterogeneous nuclear ribonucleoprotein (hnRNP) A2/B1 and osteopontin. pVHL has been characterized in vitro, however, clinical studies are limited. We evaluated the impact of $V H L$ genetic alterations on the expression of several pVHL protein targets in paired normal and tumor tissue.

Methods: The VHL gene was sequenced in 23 CCRCC patients and VHL transcript levels were evaluated by real-time RT-PCR. Expression of pVHL's protein targets were determined by Western blotting in 17 paired patient samples.

Results: VHL genetic alterations were identified in $43.5 \%$ (10/23) of CCRCCs. HIF-1 $\alpha$, HIF-2 $\alpha$ and CAIX were up-regulated in $88.2 \%(15 / 17), 100 \%(17 / 17)$ and $88.2 \%(15 / 17)$ of tumors respectively and their expression is independent of $V H L$ status. hnRNP A2/B1 and osteopontin expression was variable in CCRCCs and had no association with VHL genetic status.

Conclusion: As expression of these proposed pVHL targets can be achieved independently of VHL mutation (and possibly by hypoxia alone), these data suggests that other pVHL targets may be more crucial in renal carcinogenesis.

Keywords: Clear cell renal cell carcinoma (CCRCC), Hypoxia Inducible Factor (HIF), mutation, putative targets, von HippelLindau (VHL)
\end{abstract}

\section{Introduction}

Renal cell carcinoma (RCC) accounts for 3\% of all new cancer cases diagnosed worldwide annually. The predominant histological subtype of RCC is clear cell RCC (CCRCC). CCRCCs are typically sporadic, however, they can occur as part of a rare inherited cancer syndrome, von Hippel-Lindau (VHL) disease [21]. $V H L$ gene mutations, promoter hypermethylation and loss of heterozygosity at the $V H L$ locus are frequent occurrences in sporadic CCRCCs [14].

\footnotetext{
*Corresponding author: Sharon L. McKenna, Leslie C. Quick Laboratory, Cork Cancer Research Centre, BioSciences Institute, University College Cork and Mercy University Hospital, Cork, Ireland. Tel.: +353 21 4901333; Fax: +353 21 4901377; E-mail: s.mckenna@ucc.ie.

***Both Sharon L. McKenna and Gerald C. O'Sullivan are joint senior authors.
}

pVHL is a multi-functional tumour suppressor protein. Its best-characterised role is as the substrate recognition component of an E3 ubiquitin ligase complex which targets proteins for proteasomal degradation. Substrates of this complex include Hypoxia Inducible Factor (HIF)- $1 \alpha$ and HIF- $2 \alpha$ [11]. HIF- $\alpha$ (oxygen labile) dimerises with HIF-1 $\beta$ to form an active transcription factor complex under hypoxic conditions. HIF mediates the cell's hypoxic response by activating the transcription of numerous genes, many of which have been implicated in tumourigenesis [28]. In the absence of functional pVHL, HIF- $\alpha$ is stabilised irrespective of oxygen and constitutively activates target genes [11].

Several other proteins have been proposed as $\mathrm{pVHL}$ targets [11], including carbonic anhydrase (CA)IX, heterogeneous nuclear ribonucleoprotein ( $\mathrm{hnRNP}$ ) A2/B1 and osteopontin. The reintroduction of wild- 
type VHL into pVHL defective RCC cell lines inhibits CAIX and hnRNP A2/B1 expression [10,26]. CAIX has been associated with tumourigenesis [27]. Microarray analysis indicates that $V H L$ expression is inversely correlated with osteopontin expression [16]. Elevated tumour osteopontin expression has been associated with poor CCRCC patient prognosis [18].

Despite the extensive in vitro data on PVHL and its potential targets, no study has examined whether $V H L$ genetic alterations lead to the differential expression of several of these targets in CCRCC tissue samples. A number of studies have investigated HIF expression alone but conclusions are conflicting [7,20,30,32]. This analysis would provide important information on the clinical relevance of $V H L$ genetic alterations and establish whether therapeutic strategies directed at pVHL targets (e.g., HIF) should be restricted to patients with $V H L$ inactivation.

Therefore, we determined the VHL mutational status of 23 CCRCC patients and evaluated whether the expression of HIF- $1 \alpha$, HIF-2 $\alpha$, CAIX, hnRNP A2/B1 and osteopontin was related to the tumour's VHL status.

\section{Material and methods}

\subsection{Patient samples}

Following informed consent, paired normal renal and tumour samples were collected from 23 patients who underwent a nephrectomy procedure for RCC in the Mercy University Hospital, Cork, Ireland. The Clinical Research Ethics Committee of the Cork teaching hospitals approved this study. Following histopathological examination, tumour tissue and normal tissue (distal to the tumour) samples were immediately snap-frozen in liquid nitrogen and stored at $-80^{\circ} \mathrm{C}$. In sampling the tumour, any possible necrotic areas were avoided. Pathological analysis confirmed that all the tumour samples were classical CCRCCs. Tumours of various histological grades were included in this study.

\subsection{Stable cell line}

The 786-O cell line lacks one $V H L$ allele and has a one nucleotide deletion at Nt. 523 (codon 104) which results in a truncated VHL protein from the second allele. This cell line was obtained from the ECACC. Mutational analysis confirmed the single nucleotide deletion at codon 104. This cell line was transfected with full length human VHL (pRcCMV-HA-VHL, kindly provided by Dr. Kaelin, Boston, MA, USA) to generate 786-O VHL cells [9].

\subsection{RNA extraction and RT-PCR conditions}

RNA was extracted using the ToTally RNA extraction kit and treated with DNase (Ambion, Ireland). cDNA was synthesised using the Omniscript cDNA synthesis kit (Qiagen, UK). A $30 \mu \mathrm{l}$ PCR reaction contained $0.33 \mu \mathrm{M}$ each of sense and anti-sense primer, $1 \mu \mathrm{l}$ template cDNA and 1X Qiagen HotStarTaq Master Mix (Table 1). PCR conditions: $95^{\circ} \mathrm{C}$ for $15 \mathrm{~min}$; $(35 \times) 94^{\circ} \mathrm{C}$ for $1 \mathrm{~min}, 62^{\circ} \mathrm{C}$ for $1 \mathrm{~min}, 72^{\circ} \mathrm{C}$ for $1 \mathrm{~min}$; followed by $72^{\circ} \mathrm{C}$ for $10 \mathrm{~min}$. PCR products were sequenced by Eurofins-MWG-Operon. With these primers sets, the entire coding region of the $V H L$ mRNA was sequenced.

\subsection{Quantification of VHL transcript levels}

Real-time RT-PCR was performed using the Lightcycler system (Roche Diagnostics, UK). A $20 \mu \mathrm{l}$ reaction contained $0.5 \mu \mathrm{M}$ of each sense and anti-sense primer, $\mathrm{MgCl}_{2}$ (4 mM for VHL, $3 \mathrm{mM}$ for $\beta$-actin), $1 \mathrm{X}$ LightCycler FastStart DNA Master SYBR Green and $1 \mu \mathrm{l}$ template cDNA (Table 1). PCR conditions: $95^{\circ} \mathrm{C}$ for $10 \mathrm{~min}$; $\left(40 \times\right.$ for $\mathrm{VHL}(30 \times$ for $\beta$-actin) $) 95^{\circ} \mathrm{C}$ for $10 \mathrm{~s}, 62^{\circ} \mathrm{C}$ for $5 \mathrm{~s}$ for VHL $\left(60^{\circ} \mathrm{C}\right.$ for $\beta$-actin), $72^{\circ} \mathrm{C}$ for $5 \mathrm{~s}$ for VHL ( $16 \mathrm{~s}$ for $\beta$-actin). Melting curve: $95^{\circ} \mathrm{C}$ for $0 \mathrm{~s}, 72^{\circ} \mathrm{C}$ for $15 \mathrm{~s}\left(70^{\circ} \mathrm{C}\right.$ for $\beta$-actin $), 99^{\circ} \mathrm{C}$ for $0 \mathrm{~s}$; cooling step: $40^{\circ} \mathrm{C}$ for $30 \mathrm{~s}$. Reactions were in triplicate. Relative $V H L$ transcript levels between each

Table 1

Primer sequences used for $V H L$ mutational and transcript analysis

\begin{tabular}{ll}
\hline Primer & \multicolumn{1}{c}{ Sequence } \\
\hline VHL mRNA primer set 1 & \\
VHL197f & TCTGGATCGCGGAGGGAAT \\
VHL662r & TTGGCAAAAATAGGCTGTCC \\
VHL mRNA primer set 2 & \\
VHL555f & TCACCTTTGGCTCTTCAGA \\
VHL982r & GGTGCCTATTTTACTCTGAG \\
VHL real-time PCR & \\
primers & \\
VHL588f & CGATGGGCTTCTGGTTAACCAA \\
VHL684r & TGGAGGCATCGCTCTTTCAGA \\
$\beta$-actin & \\
$\beta$-F & \\
$\beta$-R & TTCTACAATGAGCTGCGTGTGGCT \\
\hline
\end{tabular}


of the paired patient samples was quantified using the 'Delta-Delta' mathematical formula [25].

\subsection{Western blotting}

Samples were homogenised in RIPA Buffer (50 mM Tris $\mathrm{HCl}$ ( $\mathrm{pH} 7.4$ ), $1 \%$ igepal, $0.25 \%$ sodium deoxycholate, $150 \mathrm{mM} \mathrm{NaCl}, 1 \mathrm{mM}$ EDTA, $1 \mathrm{X}$ Pefabloc, $1 \mathrm{mM} \mathrm{Na} \mathrm{VO}_{4}, 1 \mathrm{mM} \mathrm{NaF}, 1 \mathrm{X}$ protease inhibitor cocktail). Protein samples were separated by SDS-PAGE (8-12\%) and electrophoretically transferred onto nitrocellulose membrane. Primary antibodies: anti-VHL (BD Pharmingen, UK; 1:250 dilution); anti-HIF-1 $\alpha$ (BD Transduction Laboratories, UK; 1:250 dilution); anti-HIF-2 $\alpha$ (Novus Biologicals, CO, USA; 1:1000 dilution); anti-CAIX (kindly provided by Dr. Oosterwijk, University Medical Center Nijmegen, The Netherlands; 1:1000 dilution); antihnRNP A2/B1 (clone DP3B3, Sigma-Aldrich, Ireland (1:1000 dilution); clone EF67, Santa Cruz Biotechnology, UK (1:1000 dilution)); anti-osteopontin (R\&D Systems, UK; $1: 500$ dilution) and anti- $\beta$-actin (SigmaAldrich, Ireland; 1:5000 dilution). Membranes were incubated with the relevant horseradish peroxidase conjugated secondary antibodies and detected by chemiluminescence (ECL - Amersham, UK).

\subsection{Immunohistochemistry}

Paraffin embedded sections were deparaffinised and rehydrated in graded alcohol. For general histology, one section was stained by hematoxylin and eosin (H\&E) and the remaining sections were used for immunohistochemistry. The sections were subjected to antigen retrieval in $10 \mathrm{mM}$ sodium citrate buffer (pH 6.0) and a microwave treatment for $15 \mathrm{~min}$ at low power. Tissue sections were incubated in $3 \% \mathrm{H}_{2} \mathrm{O}_{2}$ for 20 min followed by washing with PBS and then blocked (5\% BSA in 1X PBS and $0.5 \%$ Tween) for $1 \mathrm{~h}$. Tissue sections were incubated overnight with HIF- $2 \alpha$ (Novus Biologicals, USA; 1:100 dilution). HIF-2 $\alpha$ expression was determined using Dako Envision+ System-HRP Kit as per manufactures' instructions.

\section{Results}

\subsection{VHL genetic alteration analysis}

Normal renal and tumour tissue from 23 CCRCC patients were screened for $V H L$ mutations. VHL mu- tations were detected in 9 patients' tumour tissues $(39.1 \%)$ (Table 2) and were absent in normal renal tissues. Mutations were located within the defined functional domains of pVHL. All mutations are predicted to cause amino acid changes and may affect pVHL's interaction with other proteins (Fig. 1A). Several tumour samples were H\&E stained, which confirms the relatively homogeneous nature of CCRCC and shows that the material sequenced was predominantly tumour tissue (Fig. 1B). There was no significant difference in the percentage of tumour cells in samples which had a VHL mutation compared to those that were classified as wild-type. In addition, it was determined that even if a tumour was $50 \%$ contaminated with normal cells, a mutation could still be identified (see Suppl. Fig. 1: http://www.qub.ac.uk/isco/JCO/). These results indicate that the assay was sensitive enough for mutational analysis in CCRCC.

There were 9 paired patient samples available for VHL transcript analysis. Four of these patients (44.4\%) had VHL transcript down-regulation in their tumour compared to the corresponding normal tissue (Fig. 1C and Table 2). VHL mutations were identified in 3 of these patients. No mutations were identified in the patients with equivalent $V H L$ transcript levels in paired tissue. Overall, $43.5 \%$ of CCRCC patients had somatic $V H L$ mutations and or VHL transcript down-regulation.

$V H L$ transcript down-regulation in 4 of 9 patients may be due to epigenetic changes or loss of heterozygosity $(\mathrm{LOH})$. Therefore it remains possible that a number of wild-type $V H L$ patients who were not amenable to this transcript analysis may have downregulation of the $V H L$ transcript. While numbers are small, it is notable that 3 out of 4 patients with transcript down-regulation also had VHL mutations. It is possible that transcript down-regulation may facilitate a requirement for bi-allelic inactivation of pVHL's tumour suppressive activity.

\subsection{HIF-1 $\alpha, H I F-2 \alpha$ and CAIX expression in paired CCRCC patient samples}

The expression levels of putative pVHL targets were analysed in paired tissue samples from 17 of the 23 patients with known VHL genetic status. Six patients were excluded due to lack of tissue. HIF- $1 \alpha$ and HIF-2 $\alpha$ were detected in the tumour tissue of $15(88.2 \%)$ and $17(100 \%)$ of CCRCC patients re- 
Table 2

Summary of $V H L$ genetic alterations identified in sporadic CCRCC patients

\begin{tabular}{|c|c|c|c|c|c|c|c|c|}
\hline $\begin{array}{l}\text { Patient } \\
\text { no. }\end{array}$ & $\begin{array}{c}\text { Fuhrman } \\
\text { grade }\end{array}$ & $\begin{array}{c}V H L \\
\text { mutation }\end{array}$ & CDS & Exon & $\begin{array}{l}\text { Codon \& predicted } \\
\text { amino acid } \\
\text { change }\end{array}$ & $\begin{array}{l}\text { Mutation } \\
\text { type }\end{array}$ & $\begin{array}{l}\text { Mutation } \\
\text { previously } \\
\text { identified }\end{array}$ & $\begin{array}{l}V H L \text { transcript } \\
\text { down-regulation }\end{array}$ \\
\hline 0 & UD & Nt. $619 \mathrm{~T} \rightarrow \mathrm{G}$ & Nt. $406 \mathrm{~T} \rightarrow \mathrm{G}$ & 2 & F136V & MS & No & Yes \\
\hline 1 & UD & Nt. $556 \mathrm{C} \rightarrow \mathrm{A}$ & Nt. $343 \mathrm{C} \rightarrow \mathrm{A}$ & 2 & $\mathrm{H} 115 \mathrm{~N}$ & MS & Yes & Yes \\
\hline 4 & III & Nt. $545 \mathrm{G} \rightarrow \mathrm{A}$ & Nt. $332 \mathrm{G} \rightarrow \mathrm{A}$ & 1 & S111N & MS & Yes & ND \\
\hline 5 & UD & $\begin{array}{c}\text { Nt. } 536-N t .550 \\
15 n t \text { deletion }\end{array}$ & $\begin{array}{c}\text { Nt. } 323-N t .337 \\
15 n t \text { deletion }\end{array}$ & 1 & $\begin{array}{l}\text { Deletion within } \\
\text { codons 108-113 }\end{array}$ & IFD \& MS & No & Yes \\
\hline & & Nt. $551 \mathrm{G} \rightarrow \mathrm{C}$ & Nt. $338 \mathrm{G} \rightarrow \mathrm{C}$ & & $\mathrm{R} 113 \mathrm{P}$ & & & \\
\hline 10 & III & Nt. $768 \mathrm{C} \rightarrow \mathrm{G}$ & Nt. $555 \mathrm{C} \rightarrow \mathrm{G}$ & 3 & Y185Stop & NS & Yes & ND \\
\hline 19 & II & - & - & - & - & - & - & Yes \\
\hline 22 & IV & $\begin{array}{c}\text { Nt. 437-Nt. } 439 \\
\text { 3nt deletion }\end{array}$ & $\begin{array}{l}\text { Nt. 224-Nt. } 226 \\
\text { 3nt deletion }\end{array}$ & 1 & $\begin{array}{l}\text { Deletion within } \\
\text { codons } 75-76\end{array}$ & IFD & Yes & ND \\
\hline 33 & III & Nt. $719 \mathrm{~T} \rightarrow \mathrm{C}$ & Nt. $506 \mathrm{~T} \rightarrow \mathrm{C}$ & 3 & L169P & MS & Yes & ND \\
\hline 40 & II & Nt. $451 \mathrm{~A} \rightarrow \mathrm{C}$ & Nt. $238 \mathrm{~A} \rightarrow \mathrm{C}$ & 1 & S80R & MS & Yes & ND \\
\hline 41 & II & $\begin{array}{c}\text { Nt. } 746-\text { Nt. } 757 \\
12 \text { nt deletion }\end{array}$ & $\begin{array}{c}\text { Nt. } 533-\text { Nt. } 544 \\
12 \text { nt deletion }\end{array}$ & 3 & $\begin{array}{l}\text { Deletion within } \\
\text { codons } 178-182\end{array}$ & IFD & No & ND \\
\hline
\end{tabular}

Notes: The entire coding region of the VHL mRNA was screened for mutations. VHL mRNA nucleotide and codon numbering is according to Genbank sequence NM_000551.2. In addition the coding sequence (CDS) nucleotide numbering is included. The published literature and the VHL mutation database (http://www. umd.be.2020) were examined to determine if these mutations had previously been reported. UD - undefined; Nt - nucleotide; MS - missense; IFD - in-frame deletion; NS - nonsense; - (dashed lines) absence of VHL mutation; ND - not determined. 


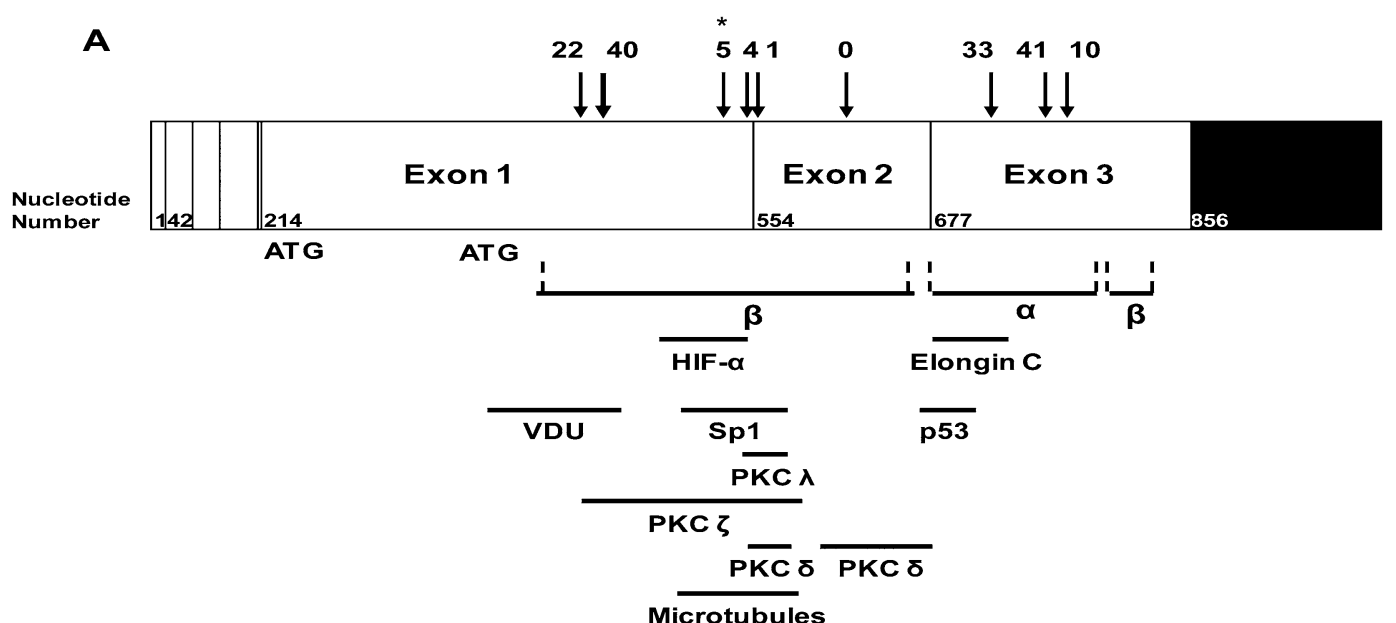

B

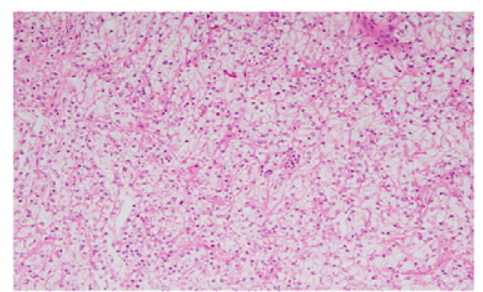

Patient 24 (no detectable VHL mutation)

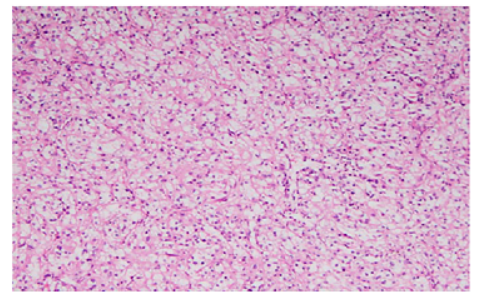

Patient 43 (no detectable VHL mutation)

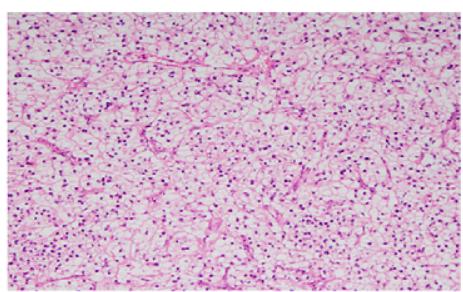

Patient 5 (VHL mutation)

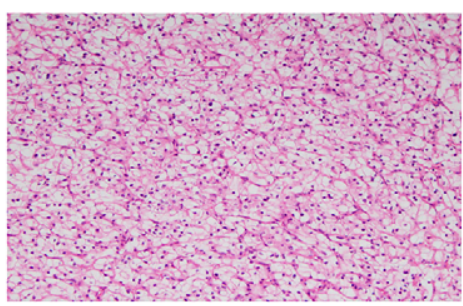

Patient 33 (VHL mutation)

Fig. 1. Schematic illustration of the location of identified $V H L$ mutations, H\&E staining of CCRCC tissue sections and $V H L$ transcript analysis in paired CCRCC tissue samples. (A) The first translation initiation codon is located at Nt. 214 with a second initiation codon at Nt. 373 (nucleotide numbering according to GenBank sequence NM_000551.2). The $5^{\prime}$ untranslated region (UTR) (hatched region) in exon 1 and the $3^{\prime}$ UTR (blackened region) in exon 3 are depicted. An arrow represents the location of each patient's mutation. Mutations were located within the defined functional domains of pVHL. The lower part of the schematic shows the location of the $\alpha$ and $\beta$ domain and the major protein binding sites of pVHL including HIF, elongin C, VDU (VHL interacting deubiquitinating enzyme), Sp1, p53, PKC $(\delta, \zeta, \lambda$ (protein kinase C)) and microtubules. (B) H\&E staining of CCRCC paraffin embedded tissue sections from two patients without detectable VHL mutations and two patients with $V H L$ mutations. (C) Relative quantification of the $V H L$ transcript level in nine patients' normal renal and tumour tissue. The 'Delta-Delta' formula was used to determine the ratio of $V H L$ transcript expression between each of the paired normal and tumour samples [25]. $\beta$-actin was used as a control reference gene. Expression Ratio $=2^{-\left(\Delta C_{\mathrm{p}} \operatorname{sample}\left(C_{\mathrm{p}} \text { Target }-C_{\mathrm{p}} \text { Reference }\right)-\Delta C_{\mathrm{p}} \text { control }\left(C_{\mathrm{p}} \text { Target }-C_{\mathrm{p}} \text { Reference }\right)\right)}$. $(2-$ real-time PCR amplification efficiency; $C_{\mathrm{p}}$ - crossing point; sample - tumour; control - corresponding normal tissue; target - VHL; reference $-\beta$-actin). For comparison purposes the expression level of $V H L$ in a normal renal tissue (control) is given a value of 1 and the expression level of $V H L$ in the corresponding tumour is compared to it. Using this formula, a two-fold down-regulation of $V H L$ would have a relative expression ratio of $<0.5$ as indicated by the dotted line (data not shown). Patient names are plotted versus the expression ratio as determined using the above formula. Error bars represent standard error of the mean. ${ }^{*}-$ two different mutations were identified in this patient's tumour tissue.

spectively and were absent in normal renal tissues (Fig. 2A, B and Table 3). Both proteins were expressed in all VHL genetically altered tumours. In the 10 pa- tients without $V H L$ genetic alterations, HIF- $1 \alpha$ and HIF- $2 \alpha$ were detected in 8 and 10 patient tumours respectively. Mean tumour diameter ranged in size from 
C VHL Transcript Analysis

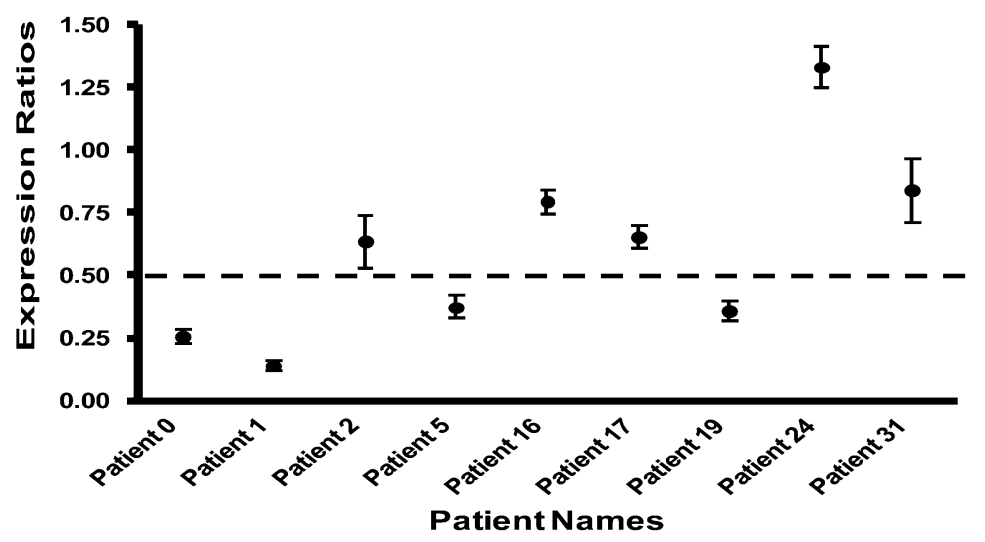

Fig. 1. (Continued.)

$3.6-18 \mathrm{~cm}$ (mean tumour size $7.6 \mathrm{~cm}$ ). HIF- $\alpha$ proteins were expressed even in the smallest tumours (data not shown). To exclude the possibility of HIF staining being primarily in stromal tissue, we evaluated HIF$2 \alpha$ expression in a number of samples by immunohistochemistry. Stromal contamination was generally low, and HIF-2 $\alpha$ expression was evident in the tumour cells irrespective of VHL mutation status (Fig. 2B(ii)). These results indicate that $V H L$ status does not predict increased expression of these proteins and that it may be achieved through hypoxia alone.

$\mathrm{pVHL}$ has also been proposed to influence CAIX expression [10]. CAIX was expressed in 15 of $17(88.2 \%)$ patient tumours (Fig. 2C and Table 3). All VHL genetically altered tumours expressed CAIX. Eight of ten wild-type $V H L$ tumours expressed CAIX suggesting that $V H L$ genetic alterations are not required for CAIX expression. Its expression may be a consequence of HIF- $\alpha$ protein expression as 13 of 17 CCRCCs expressed HIF- $1 \alpha$, HIF- $2 \alpha$ and CAIX. However, increased abundance of HIF- $\alpha$ did not always result in CAIX expression (Table 3 ).

\section{3. hnRNP A2/B1 expression in paired CCRCC patient samples}

hnRNP A2/B1 has been proposed as a pVHL E3 ubiquitin ligase complex target. Increased hnRNP A2/B1 expression was reported in the pVHL defective 786-O cell line compared to 786-O cells expressing wild-type pVHL [26]. We found however that hnRNP A2/B1 expression was equivalent in the 786-O (VHL null) cell line and 786-O clones that were transfected with VHL. Data was confirmed with an additional hnRNP A2/B1 antibody (Fig. 3A).
hnRNP A2/B1 down-regulation occurred in 11 of 17 (64.7\%) patients' tumours compared to their respective normal tissue. hnRNP A2/B1 expression was comparable in 4 patients $(23.5 \%)$ and in 2 patient $(11.8 \%)$ tumours, hnRNP A2/B1 was up-regulated (Fig. 3B, C and Table 3). hnRNP A2/B1 down-regulation was a frequent event $(6 / 7)$ in $V H L$ genetically altered tumours. Of the 10 patients with wild-type pVHL, hnRNP A2/B1 down-regulation occurred in 5 patients, while 1 patient tumour had up-regulated hnRNP A2/B1. Comparable hnRNP A2/B1 expression levels were present in 4 paired patient samples. This suggests that hnRNP A2/B1 down-regulation may occur frequently in CCRCCs independent of $V H L$ genetic alterations.

hnRNP A2/B1 down-regulation has been reported to occur in hypoxic tumour cells [6]. hnRNP A2/B1 down-regulation occurred in 10 of 15 and 11 of 17 patients that had up-regulation of HIF- $1 \alpha$ and HIF- $2 \alpha$ respectively. This indicates that hnRNP A2/B1 downregulation in these tumours may be at least in part a consequence of tumour hypoxia.

\subsection{Osteopontin expression in paired CCRCC patient samples}

Expression of osteopontin has been reported to be inversely correlated with VHL [16]. Osteopontin expression has also been shown to be up-regulated in RCCs [18]. Osteopontin was found to be up-regulated in 5 of $17(29.4 \%)$ tumours and down-regulated in $5(29.4 \%)$ tumours compared to their corresponding normal. Comparable osteopontin expression levels were detected in 7 paired patient $(41.2 \%)$ tissue 


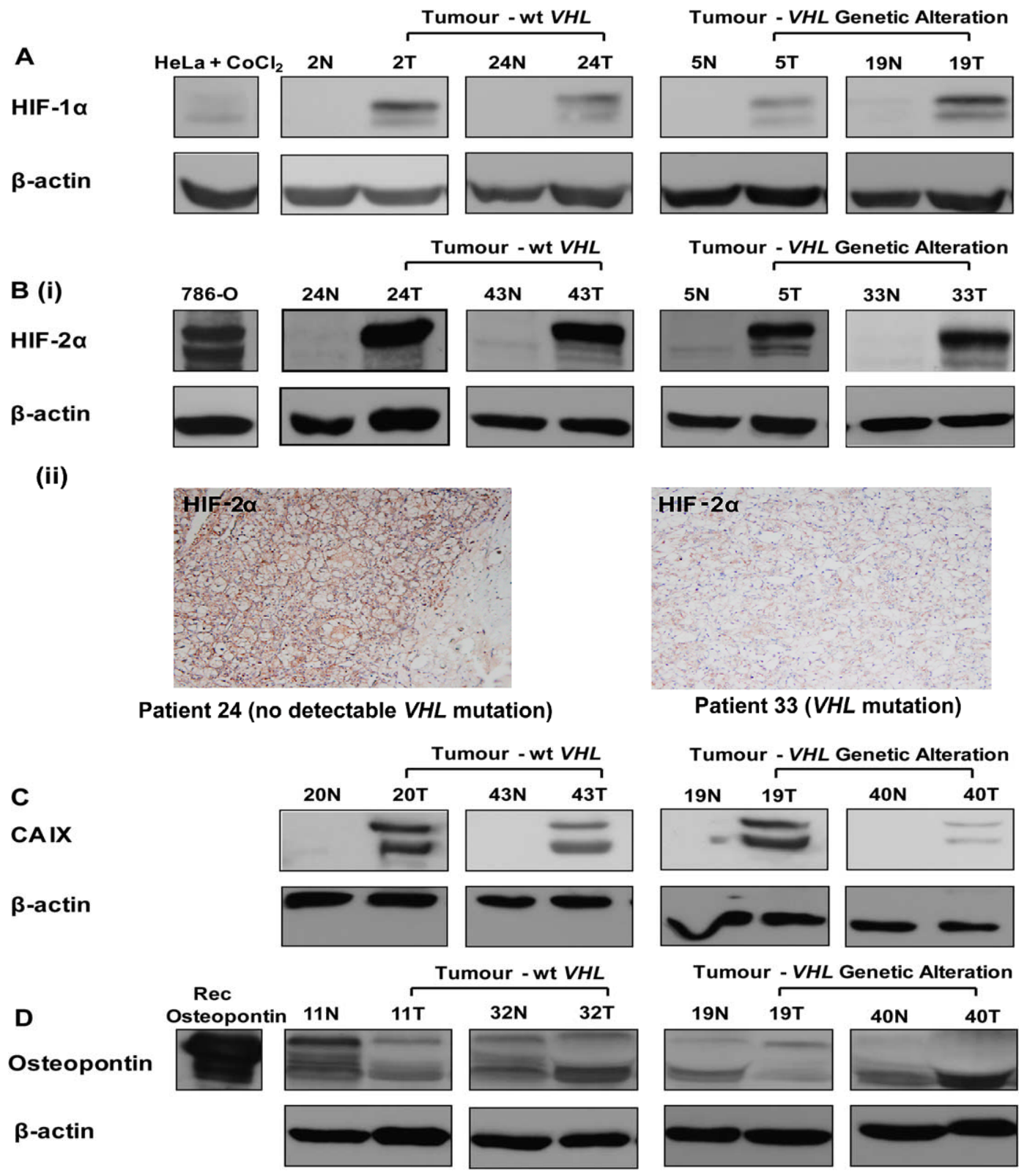

Fig. 2. HIF- $1 \alpha$, HIF-2 $\alpha$, CAIX and osteopontin expression in paired CCRCC tissue samples. Western blot analysis of HIF- $1 \alpha$ (A), HIF-2 $\alpha$ (B(i)), CAIX (C) and osteopontin (D) expression in paired normal renal and tumour tissue protein lysates. (B(ii)) Immunohistochemical analysis of HIF- $2 \alpha$ expression in CCRCC paraffin embedded tissue sections. The positive control for HIF-1 $\alpha$ is a protein lysate prepared from hypoxic HeLa cells (hypoxia induced with $75 \mu \mathrm{M} \mathrm{CoCl}_{2}$ for $16 \mathrm{~h}$ ). In the case of HIF-2 $\alpha$, the positive control is a protein lysate from the $786-\mathrm{O}$ renal cancer cell line (this is a pVHL deficient cell line which constitutively expresses HIF-2 $\alpha$ ). Osteopontin in renal tissues is expressed in the form of 72, 66 and $58 \mathrm{kDa}$ protein bands [31]. Recombinant human osteopontin (R\&D Systems) is a positive control for osteopontin. VHL genetic alterations were not detected in Patients 2,11,20, 24, 32 and 43 tumour tissues. VHL genetic alterations were identified in Patient 5, 19, 33 and 40 tumour tissues. $\beta$-actin was a loading control. $\mathrm{N}$ - normal; $\mathrm{T}-$ tumour

samples (Fig. 2D and Table 3). In 7 patients with VHL genetic alterations, up-regulation of tumour osteopontin occurred in 2 patients and 2 patients showed down-regulation of osteopontin compared to the corre- sponding normal tissue. The remaining 3 patients with mutations had equal osteopontin levels in normal and tumour tissue. Therefore, osteopontin expression was also independent of $V H L$ genetic status. 
Table 3

HIF-1 $\alpha$, HIF-2 $\alpha$, CAIX, hnRNP A2/B1 and osteopontin protein expression in CCRCC patient samples

\begin{tabular}{|c|c|c|c|c|c|c|c|}
\hline Patient no. & Fuhrman grade & $V H L$ genetic alteration & $\mathrm{HIF}-1 \alpha$ & HIF- $2 \alpha$ & CAIX & hnRNP A2/B1 & Osteopontin \\
\hline 2 & UD & ND & $\uparrow$ & $\uparrow$ & $\uparrow$ & $\leftrightarrow$ & $\uparrow$ \\
\hline \multirow[t]{2}{*}{4} & III & MS Exon 1 & $\uparrow$ & $\uparrow$ & $\uparrow$ & $\uparrow$ & $\leftrightarrow$ \\
\hline & & IFD \& MS & & & & & \\
\hline \multirow[t]{2}{*}{5} & UD & $V H L$ transcript & $\uparrow$ & $\uparrow$ & $\uparrow$ & $\downarrow$ & $\downarrow$ \\
\hline & & down-regulation & & & & & \\
\hline 11 & III & ND & $\uparrow$ & $\uparrow$ & $\uparrow$ & $\leftrightarrow$ & $\downarrow$ \\
\hline 16 & UD & ND & Nil & $\uparrow$ & $\uparrow$ & $\downarrow$ & $\uparrow$ \\
\hline \multirow[t]{2}{*}{17} & II & ND & Nil & $\uparrow$ & $\uparrow$ & $\leftrightarrow$ & $\downarrow$ \\
\hline & & Wild-type $V H L$ & & & & & \\
\hline \multirow[t]{2}{*}{19} & II & $V H L$ transcript & $\uparrow$ & $\uparrow$ & $\uparrow$ & $\downarrow$ & $\downarrow$ \\
\hline & & down-regulation & & & & & \\
\hline 20 & IV & ND & $\uparrow$ & $\uparrow$ & $\uparrow$ & $\leftrightarrow$ & $\leftrightarrow$ \\
\hline 22 & IV & IFD Exon 1 & $\uparrow$ & $\uparrow$ & $\uparrow$ & $\downarrow$ & $\uparrow$ \\
\hline 24 & II & ND & $\uparrow$ & $\uparrow$ & $\uparrow$ & $\downarrow$ & $\leftrightarrow$ \\
\hline 31 & UD & ND & $\uparrow$ & $\uparrow$ & Nil & $\downarrow$ & $\leftrightarrow$ \\
\hline 32 & I & ND & $\uparrow$ & $\uparrow$ & $\uparrow$ & $\downarrow$ & $\uparrow$ \\
\hline 33 & III & MS Exon 3 & $\uparrow$ & $\uparrow$ & $\uparrow$ & $\downarrow$ & $\leftrightarrow$ \\
\hline 34 & III & ND & $\uparrow$ & $\uparrow$ & Nil & $\downarrow$ & $\leftrightarrow$ \\
\hline 40 & II & MS Exon 1 & $\uparrow$ & $\uparrow$ & $\uparrow$ & $\downarrow$ & $\uparrow$ \\
\hline 41 & II & IFD Exon 3 & $\uparrow$ & $\uparrow$ & $\uparrow$ & $\downarrow$ & $\leftrightarrow$ \\
\hline 43 & III & ND & $\uparrow$ & $\uparrow$ & $\uparrow$ & $\uparrow$ & $\downarrow$ \\
\hline
\end{tabular}

Notes: UD - undefined; ND - not detected; $\uparrow$ - up-regulation in the tumour compared to the respective normal renal tissue; $\downarrow$ - down-regulation in the tumour compared to the respective normal renal tissue; $\leftrightarrow-$ equal expression in the normal and tumour; Nil - no detectable expression in normal renal and tumour tissue samples.

\section{Discussion}

This study investigated whether the expression of several reported pVHL protein targets is actually dependent upon de-regulation of VHL in CCRCCs. We found frequent de-regulation of HIF- $\alpha$, CAIX, hnRNP A2/B1 and osteopontin in patient samples; however, expression was found to be independent of $V H L$ status.

$V H L$ genetic alterations were identified in $43.5 \%$ of our patients. Mutations were detected in $39.1 \%$ of CCRCCs. The reported incidence of VHL gene mutations varies considerably (19.6-89.4\%) in sporadic CCRCCs $[7,13]$. However, the majority of studies report an incidence rate of between 30-60\% and our mutation rate is within this range $[1,3,14,20]$. The reason for the disparity in mutation rates is unknown as similar techniques were used in all studies. Differences in incidences are not associated with variations in ethnicity. The mutations identified in our study are likely to impair pVHL's interaction with its binding partners and inhibit its tumour suppressor abilities. Several of these mutations have previously been reported which suggests that they may be crucial for CCRCC tumouri- genesis (Table 2). In this study, analysis of VHL transcript levels was confined to 9 patients and primers will anneal to wild-type sequences in the case of a heterozygous large deletion. It is therefore possible that the incidence of $V H L$ genetic alterations in our patients could be underestimated.

Elevation of HIF-1 $\alpha$ and HIF-2 $\alpha$ expression was detected in $88.2 \%$ and $100 \%$ of CCRCCs respectively. This high frequency of expression was independent of $V H L$ status and may be achieved through hypoxia alone. In all cases following excision, tumour samples were frozen within $30 \mathrm{~min}$ in liquid nitrogen. The observed up-regulation of HIF- $\alpha$ proteins in the present study is unlikely to be caused by the variations in times prior to freezing as the paired normal and tumor tissue from the same patient were always treated identically. It has also previously been shown that storage of tissue samples ex vivo for up to $1 \mathrm{~h}$ did not induce expression or alter the abundance of HIF-1 $\alpha$ [32].

The incidence of increased expression of HIF- $\alpha$ protein in the present study is similar to two other studies that evaluated HIF- $\alpha$ expression in CCRCCs of known $V H L$ status. Turner et al. reported HIF- $1 \alpha$ and HIF- $2 \alpha$ 

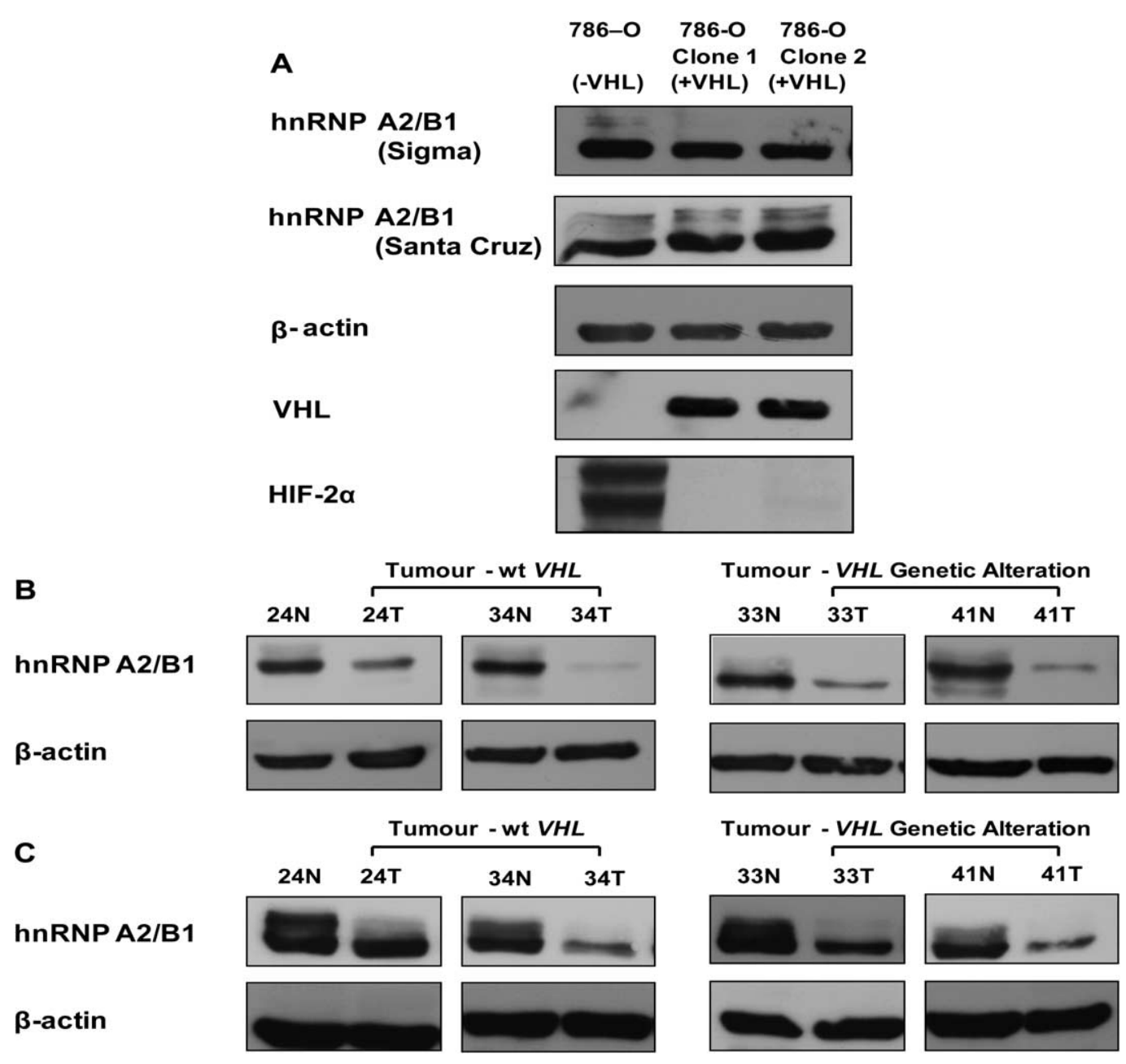

Fig. 3. hnRNP A2/B1 expression in paired normal and tumour CCRCC tissue samples. (A) Western blot analysis of hnRNP A2/B1 expression in whole cell protein lysates prepared from the 786-O cell line (VHL deficient) and two 786-O VHL cell clones (stably transfected to express pVHL) using two independent hnRNP A2/B1 antibodies. Top panel - clone DP3B3 (Sigma-Aldrich Ltd.); second panel - clone EF-67 (Santa Cruz). The blots were re-probed with anti-VHL and anti-HIF-2 $\alpha$. Western blot analysis of hnRNP A2/B1 expression in paired normal and tumour lysates using clone DP3B3 anti-hnRNP A2/B1 antibody (B), using clone EF-67 anti-hnRNP A2/B1 antibody (C). VHL genetic alterations were not detected in patients 24 and 34 tumour tissues. VHL mutations were identified in patients 33 and 41 tumour tissues.

expression in 77 and $79 \%$ of CCRCCs respectively. Up-regulation of these proteins was found to be independent of VHL mutation as $75 \%(9 / 12)$ and $50 \%$ $(6 / 12)$ of tumours without detectable mutations expressed HIF- $1 \alpha$ and HIF- $2 \alpha$ respectively [30]. Similar data were reported in a separate study of 27 CCRCCs, of which 12 (44.4\%) had VHL mutations, but HIF-1 $\alpha$ was up-regulated in $21(78 \%)$ tumours [32].

In contrast, two studies have suggested a correlation between VHL mutation and HIF- $\alpha$ expression. Analysis of 31 RCCs found that $44 \%$ had mutations but RCCs with up-regulated HIF- $1 \alpha$ were more likely to have a VHL mutation $(80 \%$ mutation rate in RCCs expressing HIF-1 $\alpha$ ) [20]. Another study re- ported an association between $V H L$ mutation and HIF de-regulation. HIF- $1 \alpha$ and HIF- $2 \alpha$ were found to be up-regulated in 61 and $88 \%$ of patients respectively. However, a very high $(89 \%)$ rate of $V H L$ inactivation was reported which will strengthen this association [7].

Expression of HIF- $\alpha$ proteins and up-regulation of pro-angiogenic factors is likely to play a major role in the progression of renal cancer. Studies of nephrectomy samples from families with VHL disease indicate that HIF- $1 \alpha$ up-regulation is detected early in discrete foci that may have bi-allellic inactivation of VHL. More advanced lesions show HIF- $2 \alpha$ up-regulation, which may be consequence of additional co-operating events [17]. The importance of HIF-2 $\alpha$ for tumour 
formation was also demonstrated in xenograft models in which HIF-2 $\alpha$ had been inactivated [15]. While these proteins are likely to be important for progression of renal and other cancers, their up-regulation may be achieved through a number of mechanisms. A recent study has reported that angiogenesis in CCRCC is not related to mutation or methylation of the $V H L$ gene [2]. Collectively, most studies indicate that HIF- $\alpha$ up-regulation is a feature of CCRCCs but that $V H L$ mutation/down-regulation does not necessarily contribute to its expression. It is possible that alterations in other $\mathrm{pVHL}$ interacting/regulating proteins could effectively cause a functional deficiency in wild-type pVHL. Mutations of the other components of the E3 ubiquitin ligase complex, elongin $\mathrm{B}$, elongin $\mathrm{C}$, Rbx 1 or the oxygen dependent degradation domain (ODDD) of HIF-1 $\alpha$ (which is the key domain for interaction with pVHL) have not been identified in CCRCCs indicating that $V H L$ represents the mutational target in this complex [4]. The current study suggests that pVHL may have other functions that contribute to carcinogenesis and HIF- $\alpha$ may play an independent but cooperative role in tumour progression.

We also evaluated CAIX expression as it is a reported pVHL target [10]. We found that $88.2 \%$ of CCRCCs expressed CAIX which is similar to previous studies $[19,24]$. CAIX up-regulation was independent of VHL status as $80 \%$ of wild-type VHL CCRCCs expressed CAIX. This result is comparable with a recent finding that $66.7 \%$ of non-VHL mutated CCRCCs expressed high CAIX levels [24]. It is likely therefore that increased CAIX expression in CCRCCs is achieved as a consequence of HIF- $1 \alpha$ expression [8, 29,33]. However in the current study, expression of HIF- $1 \alpha$ did not always predict CAIX expression.

pVHL has also been reported to down-regulate hnRNP A2/B1 [26]. We found hnRNP A2/B1 expression levels were equivalent in the 786-O cell line and two pVHL expressing 786-O clones. The reason for discrepancy between our study and Pioli et al. is unknown as similar hnRNP A2/B1 antibodies were used. In our patient cohort, hnRNP A2/B1 was down-regulated in $64.7 \%$ of CCRCCs and there was no correlation with $V H L$ status. Again, this downregulation may be a consequence of hypoxia. Hypoxia has been reported to decrease hnRNP A2/B1 transcript levels in cell lines [6]. We found an association between hnRNP A2/B1 down-regulation and upregulation of HIF- $\alpha$ proteins. However, analysis of HIF-1 $\alpha$ knockout and wild-type HIF- $1 \alpha$ fibroblasts showed that hnRNP A2/B1 expression in hypoxic con- ditions was HIF- $1 \alpha$ independent suggesting that an alternative transcription factor may cause hnRNP A2/B1 decay [6].

VHL disease patients have abnormally high plasma osteopontin levels [16]. Expression of osteopontin has been associated with renal tumour progression [18]. We found no correlation between tumour osteopontin expression and VHL status. Our incidence of tumour osteopontin expression is similar to previous studies $[18,22]$. It remains possible that osteopontin is an independent marker of progression.

The current study has found that $V H L$ inactivation occurs frequently in CCRCCs and is therefore likely to play an important role in tumourigenesis. However, HIF- $\alpha$ may not be the critical pVHL target required for CCRCC carcinogenesis as HIF de-regulation will occur irrespective of pVHL status. HIF expression is upregulated in numerous solid tumours as a consequence of tumour hypoxia [28], whereas sporadic $V H L$ loss only occurs in CCRCCs. This indicates that pVHL's role in CCRCC development may be more complex than simple HIF regulation and that it may have renal specific functions.

In contrast to in vitro studies, this analysis of clinical samples has shown that CAIX, hnRNP A2/B1 and osteopontin expression is not dependent upon $V H L$ genetic alterations. Additional functions of $\mathrm{pVHL}$ include extracellular matrix assembly, primary cilium maintenance, stabilisation of p53, Jade-1 and $\beta$-catenin regulation [23]. Animal studies also indicate that pVHL loss alone is insufficient to cause CCRCC development [12]. Recent evidence indicates that cooperative alterations of the pVHL and the PI3K pathway may be required for renal cyst development [5].

In conclusion, this study has shown that VHL mutations and or $V H L$ transcript down-regulation does not predict expression of HIF- $1 \alpha$, HIF- $2 \alpha$, CAIX, hnRNP A2/B1 and osteopontin. Our results suggest that upregulation of HIF- $\alpha$ proteins, CAIX and hnRNP A2/B1 down-regulation are common features of CCRCC and may be achieved through hypoxia alone. Therefore, further animal models and patient studies are required to fully elucidate $\mathrm{pVHL}$ 's function in CCRCC.

\section{Acknowledgements}

This work was funded by IRCSET, Cork Cancer Research Centre and the Higher Education Authority of Ireland. 


\section{References}

[1] M. Bailly, C. Bain, M.C. Favrot and M. Ozturk, Somatic mutations of von Hippel-Lindau (VHL) tumor-suppressor gene in European kidney cancers, International Journal of Cancer 63 (1995), 660-664.

[2] M.M. Baldewijns, I.J. van Vlodrop, K.M. Smits, P.B. Vermeulen, G.G. Van den Eynden, F. Schot, T. Roskams, H. van Poppel, M. van Engeland and A.P. de Bruine, Different angiogenic potential in low and high grade sporadic clear cell renal cell carcinoma is not related to alterations in the von HippelLindau gene, Cellular Oncology 31 (2009), 371-382.

[3] H. Brauch, G. Weirich, J. Brieger, D. Glavac, H. Rodl, M. Eichinger, M. Feurer, E. Weidt, C. Puranakanitstha, C. Neuhaus, S. Pomer, W. Brenner, P. Schirmacher, S. Storkel, M. Rotter, A. Masera, N. Gugeler and H.J. Decker, VHL alterations in human clear cell renal cell carcinoma: association with advanced tumor stage and a novel hot spot mutation, Cancer Research 60 (2000), 1942-1948.

[4] S.C. Clifford, D. Astuti, L. Hooper, P.H. Maxwell, P.J. Ratcliffe and E.R. Maher, The pVHL-associated SCF ubiquitin ligase complex: molecular genetic analysis of elongin $\mathrm{B}$ and $\mathrm{C}, \mathrm{Rbx} 1$ and HIF-1alpha in renal cell carcinoma, Oncogene 20 (2001), 5067-5074.

[5] I.J. Frew, C.R. Thoma, S. Georgiev, A. Minola, M. Hitz, M. Montani, H. Moch and W. Krek, pVHL and PTEN tumour suppressor proteins cooperatively suppress kidney cyst formation, The EMBO Journal 27 (2008), 1747-1757.

[6] M. Garayoa, Y.G. Man, A. Martinez, F. Cuttitta and J.L. Mulshine, Downregulation of hnRNP A2/B1 expression in tumor cells under prolonged hypoxia, American Journal of Respiratory Cell and Molecular Biology 28 (2003), 80-85.

[7] J.D. Gordan, P. Lal, V.R. Dondeti, R. Letrero, K.N. Parekh, C.E. Oquendo, R.A. Greenberg, K.T. Flaherty, W.K. Rathmell, B. Keith, M.C. Simon and K.L. Nathanson, HIF-alpha effects on c-Myc distinguish two subtypes of sporadic VHL-deficient clear cell renal carcinoma, Cancer Cell 14 (2008), 435-446.

[8] K. Grabmaier, M.C.A. de Weijert, G.W. Verhaegh, J.A. Schalken and E. Oosterwijk, Strict regulation of CAIX(G250/ $\mathrm{MN})$ by HIF-1alpha in clear cell renal cell carcinoma, Oncogene 23 (2004), 5624-5631.

[9] O. Iliopoulos, A. Kibel, S. Gray and W.G. Kaelin Jr., Tumour suppression by the human von Hippel-Lindau gene product, Nature Medicine 1 (1995), 822-826.

[10] S.V. Ivanov, I. Kuzmin, M.H. Wei, S. Pack, L. Geil, B.E. Johnson, E.J. Stanbridge and M.I. Lerman, Down-regulation of transmembrane carbonic anhydrases in renal cell carcinoma cell lines by wild-type von Hippel-Lindau transgenes, Proceedings of the National Academy of Sciences of the United States of America 95 (1998), 12596-12601.

[11] W.G. Kaelin Jr., The von Hippel-Lindau tumour suppressor protein: O2 sensing and cancer, Nature Reviews Cancer 8 (2008), 865-873.

[12] P.P. Kapitsinou and V.H. Haase, The VHL tumor suppressor and HIF: insights from genetic studies in mice, Cell Death and Differentiation 15 (2008), 650-659.

[13] J.H. Kim, C.W. Jung, Y.H. Cho, J. Lee, S.H. Lee, H.Y. Kim, J. Park, J.O. Park, K. Kim, W.S. Kim, Y.S. Park, Y.H. Im, W.K. Kang and K. Park, Somatic VHL alteration and its impact on prognosis in patients with clear cell renal cell carcinoma, Oncology Reports 13 (2005), 859-864.

[14] W.Y. Kim and W.G. Kaelin, Role of VHL gene mutation in human cancer, Journal of Clinical Oncology 22 (2004), 49915004.

[15] K. Kondo, W.Y. Kim, M. Lechpammer and W.G. Kaelin Jr., Inhibition of HIF2alpha is sufficient to suppress pVHL-defective tumor growth, PLoS Biology 1 (2003), E83.

[16] Q.T. Le, P.D. Sutphin, S. Raychaudhuri, S.C. Yu, D.J. Terris, H.S. Lin, B. Lum, H.A. Pinto, A.C. Koong and A.J. Giaccia, Identification of osteopontin as a prognostic plasma marker for head and neck squamous cell carcinomas, Clinical Cancer Research 9 (2003), 59-67.

[17] S.J. Mandriota, K.J. Turner, D.R. Davies, P.G. Murray, N.V. Morgan, H.M. Sowter, C.C. Wykoff, E.R. Maher, A.L. Harris, P.J. Ratcliffe and P.H. Maxwell, HIF activation identifies early lesions in VHL kidneys: evidence for site-specific tumor suppressor function in the nephron, Cancer Cell 1 (2002), 459468.

[18] K. Matusan, G. Dordevic, D. Stipic, V. Mozetic and K. Lucin, Osteopontin expression correlates with prognostic variables and survival in clear cell renal cell carcinoma, Journal of Surgical Oncology 94 (2006), 325-331.

[19] Y. Murakami, K. Kanda, M. Tsuji, H. Kanayama and S. Kagawa, MN/CA9 gene expression as a potential biomarker in renal cell carcinoma, British Journal of Urology International 83 (1999), 743-747.

[20] X. Na, G. Wu, C.K. Ryan, S.R. Schoen, P.A. di'Santagnese and E.M. Messing, Overproduction of vascular endothelial growth factor related to von Hippel-Lindau tumor suppressor gene mutations and hypoxia-inducible factor- 1 alpha expression in renal cell carcinomas, The Journal of Urology 170 (2003), 588-592.

[21] E.C. Nelson, C.P. Evans and P.N. Lara Jr., Renal cell carcinoma: current status and emerging therapies, Cancer Treatment Reviews 33 (2007), 299-313.

[22] A. Nishie, K. Masuda, M. Otsubo, T. Migita, M. Tsuneyoshi, K. Kohno, T. Shuin, S. Naito, M. Ono and M. Kuwano, High expression of the Cap43 gene in infiltrating macrophages of human renal cell carcinomas, Clinical Cancer Research 7 (2001), 2145-2151.

[23] M.J. Nyhan, G.C. O'Sullivan and S.L. McKenna, Role of the VHL (von Hippel-Lindau) gene in renal cancer: a multifunctional tumour suppressor, Biochemical Society Transactions 36 (2008), 472-478.

[24] J.J. Patard, P. Fergelot, P.I. Karakiewicz, T. Klatte, Q.D. Trinh, N. Rioux-Leclercq, J.W. Said, A.S. Belldegrun and A.J. Pantuck, Low CAIX expression and absence of VHL gene mutation are associated with tumor aggressiveness and poor survival of clear cell renal cell carcinoma, International Journal of Cancer 123 (2008), 395-400.

[25] M.W. Pfaffl, A new mathematical model for relative quantification in real-time RT-PCR, Nucleic Acids Research 29 (2001), e45.

[26] P.A. Pioli and W.F. Rigby, The von Hippel-Lindau protein interacts with heteronuclear ribonucleoprotein a2 and regulates its expression, The Journal of Biological Chemistry 276 (2001), 40346-40352.

[27] J.M. Purkerson and G.J. Schwartz, The role of carbonic anhydrases in renal physiology, Kidney International 71 (2007), 103-115. 
[28] E.B. Rankin and A.J. Giaccia, The role of hypoxia-inducible factors in tumorigenesis, Cell Death and Differentiation 15 (2008), 678-685.

[29] R.R. Raval, K.W. Lau, M.G. Tran, H.M. Sowter, S.J. Mandriota, J.L. Li, C.W. Pugh, P.H. Maxwell, A.L. Harris and P.J. Ratcliffe, Contrasting properties of hypoxia-inducible factor 1 (HIF-1) and HIF-2 in von Hippel-Lindau-associated renal cell carcinoma, Molecular and Cellular Biology 25 (2005), 56755686.

[30] K.J. Turner, J.W. Moore, A. Jones, C.F. Taylor, D. CuthbertHeavens, C. Han, R.D. Leek, K.C. Gatter, P.H. Maxwell, P.J. Ratcliffe, D. Cranston and A.L. Harris, Expression of hypoxia-inducible factors in human renal cancer: relationship to angiogenesis and to the von Hippel-Lindau gene mutation, Cancer Research 62 (2002), 2957-2961.

[31] A. Verhulst, V.P. Persy, A.R. Van Rompay, W.A. Verstrepen, M.F. Helbert and M.E. De Broe, Osteopontin synthesis and lo- calization along the human nephron, Journal of the American Society of Nephrology 13 (2002), 1210-1218.

[32] M.S. Wiesener, P.M. Munchenhagen, I. Berger, N.V. Morgan, J. Roigas, A. Schwiertz, J.S. Jurgensen, G. Gruber, P.H. Maxwell, S.A. Loning, U. Frei, E.R. Maher, H.J. Grone and K.U. Eckardt, Constitutive activation of hypoxia-inducible genes related to overexpression of hypoxia-inducible factor1alpha in clear cell renal carcinomas, Cancer Research 61 (2001), 5215-5222.

[33] C.C. Wykoff, N.J. Beasley, P.H. Watson, K.J. Turner, J. Pastorek, A. Sibtain, G.D. Wilson, H. Turley, K.L. Talks, P.H. Maxwell, C.W. Pugh, P.J. Ratcliffe and A.L. Harris, Hypoxiainducible expression of tumor-associated carbonic anhydrases, Cancer Research 60 (2000), 7075-7083. 


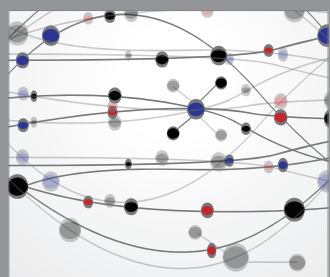

The Scientific World Journal
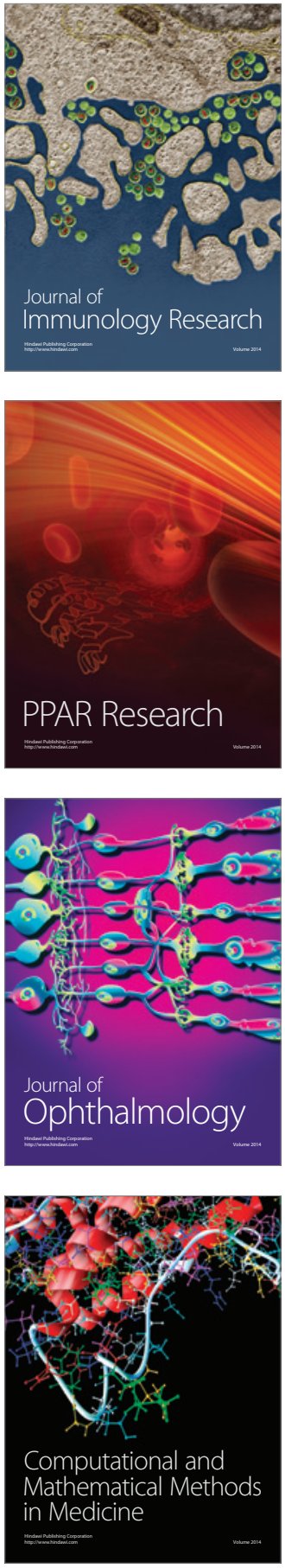

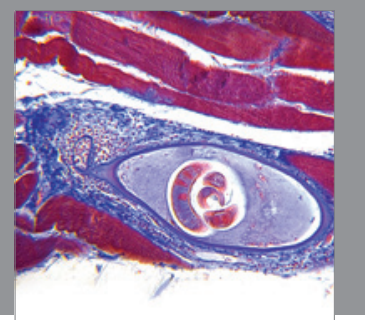

Gastroenterology

Research and Practice
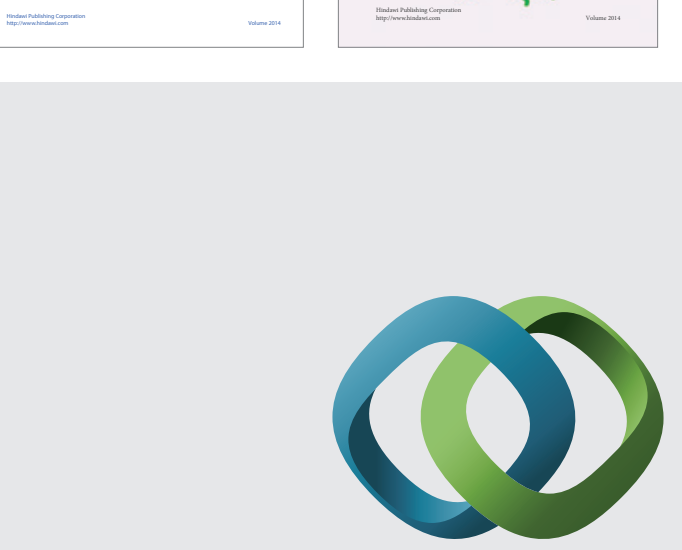

\section{Hindawi}

Submit your manuscripts at

http://www.hindawi.com
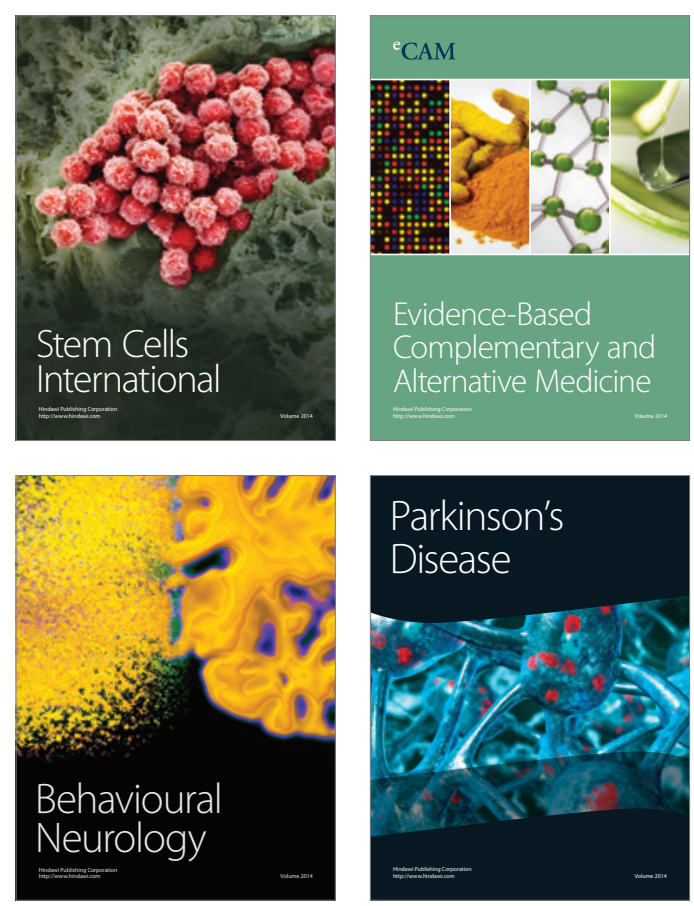

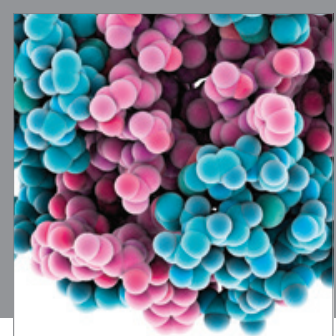

Journal of
Diabetes Research

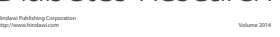

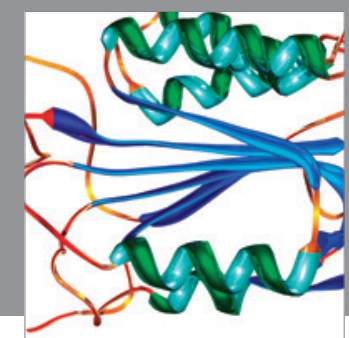

Disease Markers
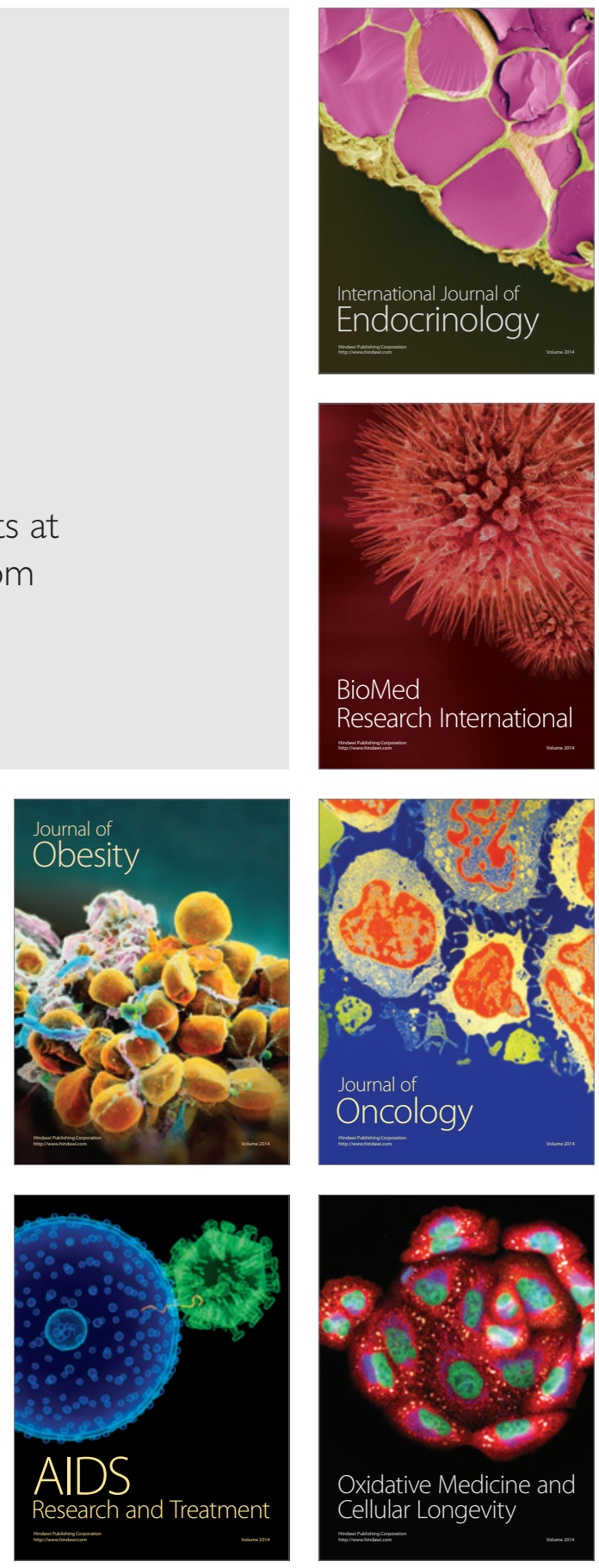